\title{
N-Methyl-D-aspartate receptor antibody could be a cause of catatonic symptoms in psychiatric patients: case reports and methods for detection
}

This article was published in the following Dove Press journal:

Neuropsychiatric Disease and Treatment

8 February 2017

Number of times this article has been viewed

\author{
Ko Tsutsui' \\ Takashi Kanbayashi',2 \\ Manabu Takaki ${ }^{3}$ \\ Yuki Omori' \\ Yumiko Imai ${ }^{4}$ \\ Seiji Nishino ${ }^{5}$ \\ Keiko Tanaka ${ }^{6}$ \\ Tetsuo Shimizu',2 \\ 'Department of Neuropsychiatry, \\ Akita University Graduate School \\ of Medicine, Akita, ${ }^{2}$ International \\ Institute for Integrative Sleep \\ Medicine (WPI-IIIS), University of \\ Tsukuba, Tsukuba, ${ }^{3}$ Department of \\ Neuropsychiatry, Okayama University, \\ Okayama, ${ }^{4}$ Biological Informatics \\ and Experimental Therapeutics, \\ Akita University Graduate School \\ of Medicine, Akita, Japan; ${ }^{5}$ Sleep and \\ Circadian Neurobiology Laboratory, \\ Stanford University School of \\ Medicine, Palo Alto, California, USA; \\ ${ }^{6}$ Brain Research Institute, Niigata \\ University, Niigata, Japan
}

\begin{abstract}
The symptoms of catatonia have been reported to be similar to the initial symptoms of anti-N-methyl-D-aspartate receptor (NMDAR) encephalitis. Subsequently, this autoimmune limbic encephalitis has been noticed by many psychiatrists. For a differential diagnosis of catatonic state, it is important to detect anti-NMDAR encephalitis. This encephalitis is expected to be in remission by early detection and treatment. We should be more cautious about catatonic symptoms of schizophrenia. When a patient is suspected of having encephalitis, we should screen for anti-NMDAR antibodies in cerebrospinal fluid samples using a cell-based assay. We describe the methods of NMDAR antibody detection and the etiology of this encephalitis with case reports. Two representative cases with catatonia and non-catatonia (brief psychotic disorder) were reported. Schizophrenia is a general, heterogeneous, and complicated disorder, and its pathophysiology is unknown. There is an established evidence of NMDAR hypofunction, which is the functional disconnection of the central component; this is one of the most recognized models for schizophrenia. Furthermore, it is said that autoimmune mechanisms have been involved, at least in subgroups of schizophrenia patients. Further study of anti-NMDAR antibody and its related encephalitis would give essential clues for the research of schizophrenia, catatonia, and atypical psychosis.
\end{abstract}

Keywords: NMDA receptor, encephalitis, antibody, psychosis, catatonia

\section{Introduction}

Recently, anti-N-methyl-D-aspartate receptor (NMDAR) encephalitis has been found to evoke psychotic states such as bizarre behavior, disorientation, confusion, paranoid thoughts, visual or auditory hallucinations, catatonia, and memory deficits. Subsequently, patients develop seizures, autonomic instability, dyskinesias, or a decreased level of consciousness. ${ }^{1-3}$ These symptoms often occur in young females with ovarian teratomas, who have shown good responses to tumor surgery and immunotherapy. ${ }^{1,4,5}$ They have also been detected with cerebrospinal fluid (CSF) and serum autoantibodies to the NMDAR. ${ }^{1}$ In Japan, clinical course of acute juvenile non-herpetic encephalitis was very similar to that of anti-NMDAR encephalitis. ${ }^{6}$ It had been recognized that these two diseases were the same. Moreover, clinical course of malignant catatonia was accompanied by symptoms, such as autonomic instability, neurological symptoms, hyperthermia, and disturbance of consciousness. Much attention has been paid to anti-NMDAR encephalitis as a catatonic differential diagnosis. This condition was also considered to be the cause of malignant or lethal catatonia. ${ }^{7}$ In addition, from a longitudinal viewpoint, this disease would be related to atypical psychosis, which was proposed by Mitsuda in Japan. ${ }^{8}$ The diagnosis of anti-NMDAR encephalitis is only
Correspondence: Takashi Kanbayashi Department of Neuropsychiatry, Akita University School of Medicine, I-I-I Hondo, Akita 010-8543, Japan

Tel +81188846122

Fax $+81 \quad 188846445$

Email takashik@med.akita-u.ac.jp (c)
hereby accept the Terms. Non-commercial uses of the work are permitted without any further permission from Dove Medical Press Limited, provided the work is properly attributed. For permission for commercial use of this work, please see paragraphs 4.2 and 5 of our Terms (https://www.dovepress.com/terms.php). 
possible by the detection of anti-NMDAR antibodies ( $\operatorname{IgG}$ ), which is specific for anti-NMDAR encephalitis.

\section{NMDAR}

NMDARs are located in the post-synaptic membrane, and are glutamate receptors consisting of GluN1 subunits and two GluN2/3 subunits. ${ }^{9} 10$ There are eight alternatively spliced GluN1 isoforms, two GluN3 subunits (A-B), and four GluN2 subunits (A-D). The NMDAR is expressed at the cell surface when both of GluN1 and GluN2/3 exist and form a tetrameric structure. Glutamate hypothesis of schizophrenia is well known to psychiatrists. ${ }^{11}$ Dysfunction of the NMDAR provokes lowered glutamic transmission, which leads to many psychotic symptoms. Anti-NMDAR encephalitis is thought to be a disease caused by the antibodies that recognize receptor protein expressed on the cell membrane surface and results in functional damage.

\section{The pathology of anti-NMDAR encephalitis}

The antibodies from patients with anti-NMDAR encephalitis bind to the cell surface receptor clusters, and cause an internalization of the receptors and decrease the NMDAR-related membrane current. Hughes proposed that anti-NMMDAR antibodies selectively decrease synaptic NMDAR currents and decrease NMDAR cluster density in vivo. ${ }^{12}$ It has been recognized that these antibodies cause an immune-mediated neuronal dysfunction without irreversible degeneration. Dalmau et al emphasized that these antibodies lead to the rapid and reversible loss of surface NMDARs by antibodymediated capping and internalization. In addition, it leads to the functional abrogation of NMDAR. ${ }^{13}$ Conventional co-expression of NR1 and NR2B is used for the measurement of anti-NMDAR antibody. Gleichman et al showed that the extracellular epitope of GluN1 is crucial for the immune-reactivity. ${ }^{14}$ In this study, we have described the methods of NMDAR antibody detection and the etiology of this encephalitis with case reports.

\section{Subjects and methods Subjects}

Two cases were diagnosed with catatonia or brief psychotic disorder, respectively. These cases were treated in Akita University Hospital, and the NMDAR antibody was measured in Kansai Medical University (KMU). These patients gave informed consent to lumber puncture and antibody examination, and also for their cases to be published. This study was approved by ethical committees of Akita University and KMU.

\section{Methods for anti-NMDAR antibodies detection}

GluN1 and GluN2 co-transfected cells have been used for anti-NMDAR antibody measurement (tetramer cell-based assay [CBA]; Figure 1). ${ }^{15}$

The NMDAR subunit complementary DNAs (cDNAs; GluN1 alone or GluN1 with GluN2B as an equimolar mixture $)^{16}$ were transfected with a lipofectamine reagent (Invitrogen, Japan, Tokyo) into human embryonic kidney (HEK) 293 cells as describedbelow. ${ }^{17}$ Cell culture was maintained in Dulbecco's Modified Eagle's Medium with $10 \%$ fetal bovine serum (FBS), which also contained 1 mM MK-801 (Wako, Tokyo, Japan) for neuroprotection. Twenty-four hours after transfection, HEK cells were fixed in $4 \%$ paraformaldehyde in $0.1 \mathrm{M}$ phosphate-buffered saline (PBS, pH 7.4) for 10 minutes. Non-specific binding was blocked with $20 \%$ goat serum/PBS, and the cells were incubated with patient's CSF (1:2) in $0.02 \%$ Triton X-100, $10 \%$ goat serum in PBS overnight at $4{ }^{\circ} \mathrm{C}$, and then with fluorescein isothiocyanate (FITC)-conjugated anti-human IgG (DAKO, Glostrup, Denmark; 1:50) for 30 minutes. To confirm the localization of NMDAR antibody binding sites, double staining was performed using both the patient's CSF and rabbit anti-GluN1 antibodies (PhosphoSolutions, Aurora, CO, USA; 1:10) as the primary antibodies. A mixture of FITC-conjugated anti-human IgG and PE-conjugated anti-rabbit IgG as the second antibodies was used to confirm that the patient's CSF really bind to GluN1. The cells showing an anti-NMDAR antibody-specific staining pattern under fluorescence microscope $(\times 20)$ was determined as positive (Figure 2).

\section{Results \\ Case reports}

We have studied two representative cases with psychiatric symptoms and NMDAR antibody positive. These cases had been treated in Akita University Hospital, and their antibodies were detected in KMU.

\section{Case I catatonia case, 24 year old male}

Three years before, he (case 1) had an episode of catatonia and was hospitalized in a mental hospital. Few days later, he had headache, fever, and electroencephalography (EEG) abnormality (diffuse theta wave). Thereafter, he was transferred to a general hospital. His CSF showed pleocytosis, but brain magnetic resonance imaging (MRI) and other examinations were normal. He had been under symptomatic treatment; however, he had a convulsion. Thereafter, he was 


\section{Antibody measurement method}

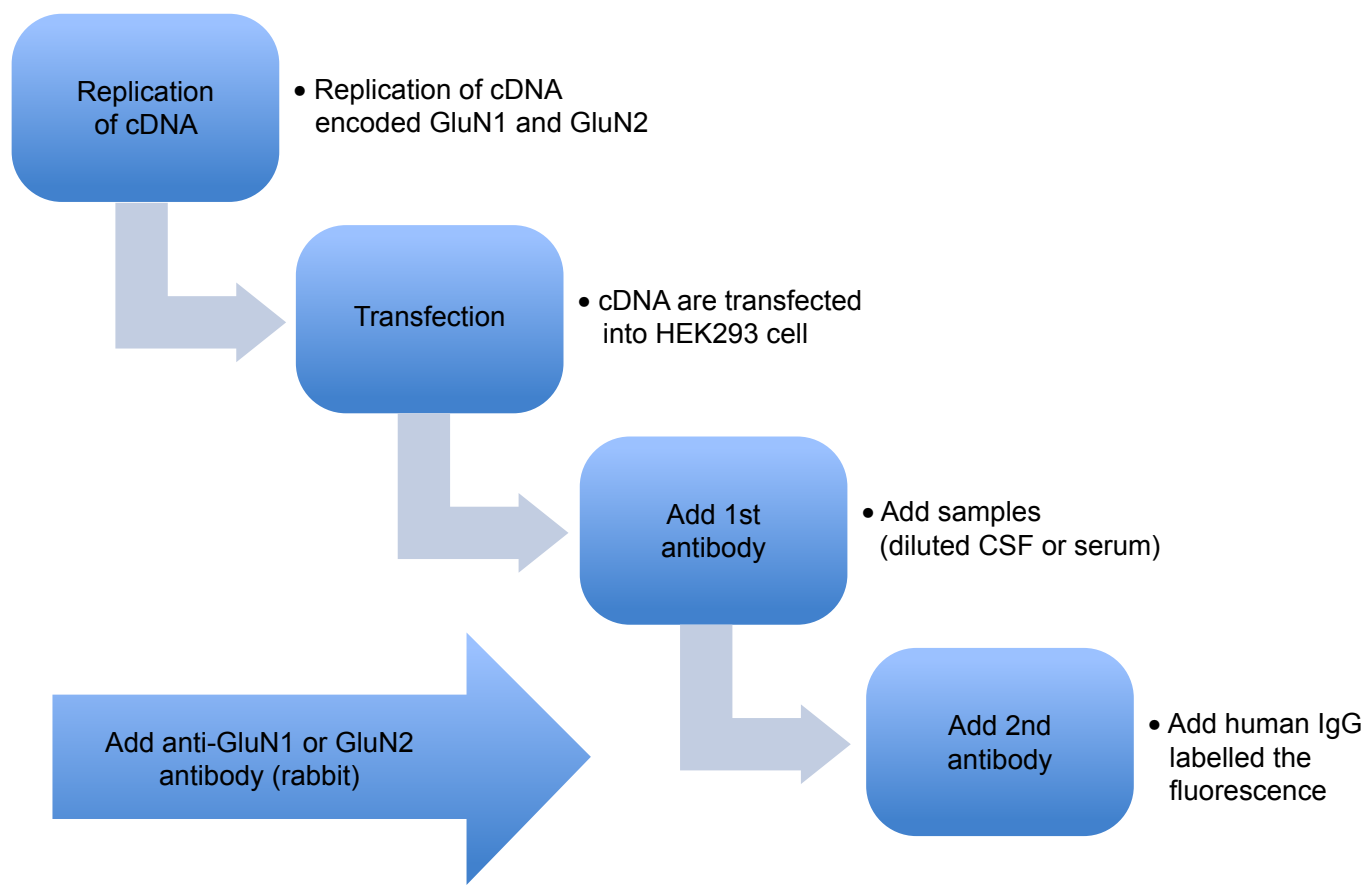

Figure I Methods of anti-NMDAR antibodies detection.

Notes: The NMDAR subunit cDNAs (GluNI alone or GluNI together with GluN2B as an eqimolar mixture) were transfected with a lipofectamine reagent into human embryonic kidney (HEK) 293 cells. The cells were incubated with patient's CSF, and then with FITC-conjugated anti-human IgG. To confirm the localization of NMDAR antibody binding sites, double staining was performed using both of the patients' CSF and rabbit anti-GluNI antibodies as the primary antibodies and a mixture of FITCconjugated anti-human IgG and PE-conjugated anti-rabbit IgG as the second antibodies to confirm that the patient CSF really bind to GluNI.

Abbreviations: cDNAs, complementary DNA; CSF, cerebrospinal fluid; FITC, fluorescein isothiocyanate; NMDAR, N-methyl-D-aspartate receptor.

treated with anticonvulsant (phenitoin), which showed good outcome. He was discharged after one month; however he stopped going to the clinic soon. Four years later, he had headache and abnormal visual field. As his headache and fever were getting worse, he was again admitted to a general hospital. His CSF showed pleocytosis, but brain MRI and other examinations were normal. Thereafter, he had restlessness, substupor, and convulsion. Since he was diagnosed with infectious encephalitis, he was treated with intravenous immunoglobulins (IVIG) and anticonvulsant (phenitoin). He did not have more convulsions; however, his psychiatric symptoms remained, such as wondering, silly smiling, and catatonia. He was also treated with steroid pulse therapy with some effects. He was transferred to a psychiatric unit for further treatment. His symptoms include disorientation, palilalia, suicide idea, self-injury, wondering, catalepsy

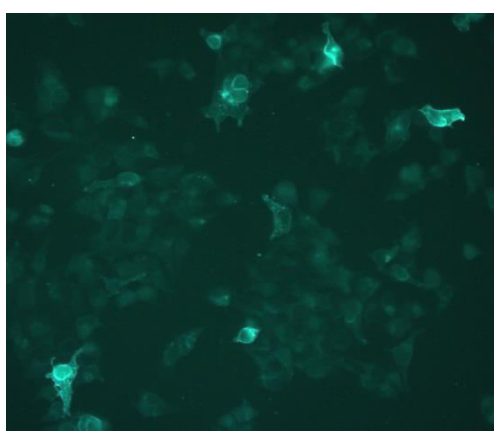

Pt. CSF-FITC anti-human IgG

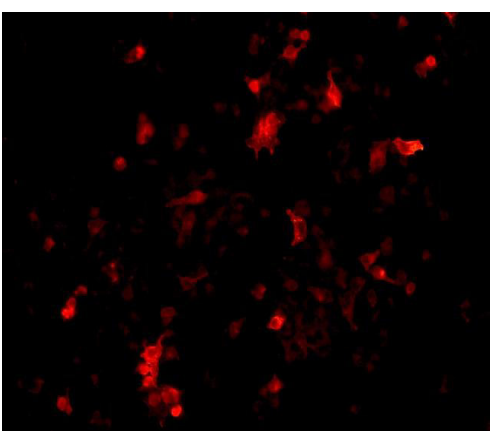

Anti-GluN1-PE-anti-rabbit lgG

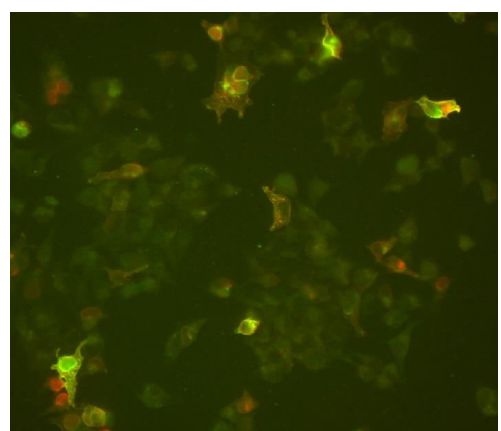

Merged

Figure 2 HEK 293 cells expressing GluNI/2 with fluorescence microscope.

Notes: HEK 293 cells expressing GluNI/2, showing reactivity with patients' antibodies (left, green), a monoclonal antibody (middle, red), and the merged reactivities (right, yellow). The cells showing anti-NMDAR antibody-specific staining pattern with fluorescence microscope $(\times 20)$ was determined as positive. "Pt" is a patient with NMDAR encephalitis.

Abbreviations: HEK, human embryonic kidney; NMDAR, N-methyl-D-aspartate receptor. 
without autonomic failure, and involuntary movements. He was treated with risperidone and levomepromazine. His symptoms were not initially changed, but few weeks later, his condition gradually got better. He was discharged without deficit two months later. His CSF showed anti-NMDAR IgG positive after his discharge.

\section{Case 2 non-catatonia case, 60 year old female}

Eight years before the first episode, she (case 2) took the operation for the ovarian cyst without sequelae. When she was 60 years old, she was confused gradually two weeks after she caught a cold, and she screamed because of auditory hallucination and delusion. She was admitted in a psychiatric unit and was given $4 \mathrm{mg}$ of risperidone. She completely recovered the next day without deficit. Her CSF showed pleocytosis, but brain MRI, EEG, and whole-body computerized tomography examinations were normal. Risperidone was reduced to $1 \mathrm{mg}$, but her psychiatric symptoms were not relapsed. She was discharged after one month. Her CSF showed anti-NMDAR IgG positive the next month. She continued $1 \mathrm{mg}$ risperidone as outpatient for one year but discontinued. After three months, she had a relapse of auditory hallucination, delusion, and restlessness. Again she was admitted, and the treatment was restarted with $2 \mathrm{mg}$ risperidone. Her psychiatric manifestations were fully recovered in a few days.

\section{Discussion}

Recently, a wide range of autoantibodies are known to relate to autoimmune neurological disorders as the diagnostic and therapeutic markers. Some of them affected directly the pathomechanisms of neurological diseases. Several autoantibodies with such roles have been reported showing the common characters as recognizing cell surface antigens. Among them, anti-NMDAR antibody in non-herpetic limbic encephalitis has drawn considerable attention. ${ }^{17,18}$ The NMDAR encephalitis is seen in young women having ovarian teratoma. This encephalitis showed memory and consciousness disturbances, agitation, psychosis, epilepsy, respiratory failure, autonomic disorders, and involuntary movements. This condition was also seen in patients with catatonic symptoms among psychiatric disorders. ${ }^{7,19}$

\section{Catatonia in DSM-5}

According to DSM-5, which was published on May 18, 2013, catatonia became an independent diagnostic category, which was associated with another mental disorder, such as mood disorders or schizophrenia, another unspecified, medical condition. In up to $25 \%$ of cases, catatonia is related with general medical or neurologic conditions. ${ }^{20,21}$ Recently, it was shown repeatedly that catatonic symptoms are observable in most patients diagnosed with anti-NMDAR encephalitis. ${ }^{22,23}$ We classified the antibody positive cases as follows: a) catatonia group, case 1 and b) non-catatonia group (brief psychotic disorder), case 2 in the current study. The catatonic symptoms were also included in the criteria of brief psychotic disorder of DSM-5. ${ }^{24}$ However, three or more catatonic symptoms were needed for catatonia diagnosis. If the patients had two catatonic symptoms, the diagnosis would be brief psychotic disorder.

\section{Cases consideration}

The first case was initially diagnosed as encephalitis with unknown etiology. He showed both neurological symptoms, such as convulsion and EEG abnormality, and catatonic symptoms, such as catalepsy, stupor, and palilalia. His symptoms would also be diagnosed as catatonia due to NMDAR encephalitis in DSM-5, and he was re-categorized as catatonia group in this study.

The episodes of case 2 fulfilled the criteria of brief psychotic disorder and exhibited fulminant delusion, hallucination, and agitation. Her symptoms improved in about a week without deficits both the times. Her two episodes had catatonic symptoms but they did not fulfill the diagnostic criteria for catatonia in DSM-5. She would also be diagnosed with atypical psychosis in the traditional diagnostic concept in Japan. ${ }^{8}$

During acute phases of brief psychotic disorder or catatonia, we had difficulty in obtaining CSF samples. If a patient has been suspected with psychiatric manifestation related to anti-NMDAR antibodies, we should examine patient's CSF.

Antibodies, such as anti-Hu, anti-Yo, anti-voltage-gated potassium channels, anti-MaTa, CV2, and antiamphiphysin in limbic encephalitis with psychosis, were reported. ${ }^{25}$ However, our two cases were not typical for the symptoms of these encephalitis, therefore we did not examine these antibodies.

\section{Atypical psychosis}

The features of the second patient are similar to those of "Atypical psychosis" proposed by Mitsuda in Japan, a notion derived from "Cycloid psychosis" conceptualized by German psychiatrist, Leonhard. Both cycloid and atypical psychoses have coinciding features of acute onset, emotional disturbances, psychomotor disturbances, alternations of consciousness, high 
prevalence in women, and oriented premorbid personality. Mitsuda had suspected involvements of brain organic changes in atypical psychosis. ${ }^{8}$ If this antibody is a cause of atypical psychosis, the speculation of Mitsuda would be proved.

\section{CSF examination}

It is not easy for psychiatrists to obtain the CSF samples. But the most optimal results are provided when we use CSF. Viaccoz et al indicated a 3\% false-positivity rate of serum testing using serum with CBA. ${ }^{26}$ Gresa-Arribas et al postulated that NMDAR antibodies always present in CSF but $13.2 \%$ of the patients do not have serum antibodies detectable with $\mathrm{CBA}^{27}$ Considering the above-mentioned reports, we used CSF and CBA for the antibody detection. The suitable candidates were schizophrenic patients with catatonic symptoms and atypical psychosis. The specific symptoms for NMDAR encephalitis were not remarkable, but lymphocytic pleocytosis in CSF were reported. ${ }^{13}$ It is said that the cost would be $\$ 50$ in the future $;^{28}$ however, presently the cost sending to a clinical laboratory is $\$ 180$ in Japan. Patients will have some somatic symptoms along with psychiatric symptoms.

\section{Treatments}

Although not currently recommended, psychiatrists would consider screening CSF for anti-NMDAR antibodies in patients presenting with first-episode psychosis, particularly in young females also presenting with catatonia. ${ }^{29-31}$ Identifying anti-NMDAR antibodies could alter the treatment rather dramatically as antipsychotics, which are the first-line treatment for psychosis, have had equivocal results, and had sometimes been harmful (described below) in cases of antiNMDAR encephalitis. ${ }^{31,32}$

Once the diagnosis of anti-NMDAR encephalitis is confirmed by the presence of autoantibodies, prompt treatment is imperative. The initial phase of treatment involves removal of a teratoma, treatment with corticosteroids, IVIGs, or plasma exchange, which is followed by treatment with rituximab or cyclophosphamide in patients who do not respond. ${ }^{13,31}$

Owing to the prominent psychiatric symptoms, patients have also been frequently administered with antipsychotics. However, antipsychotics may further confuse these complex clinical pictures by leading to the development of autonomic instability, elevated creatinine kinase, and rigidity, which are characteristics of both malignant catatonia and neuroleptic

\section{Glutamate hypothesis of schizohrenia}

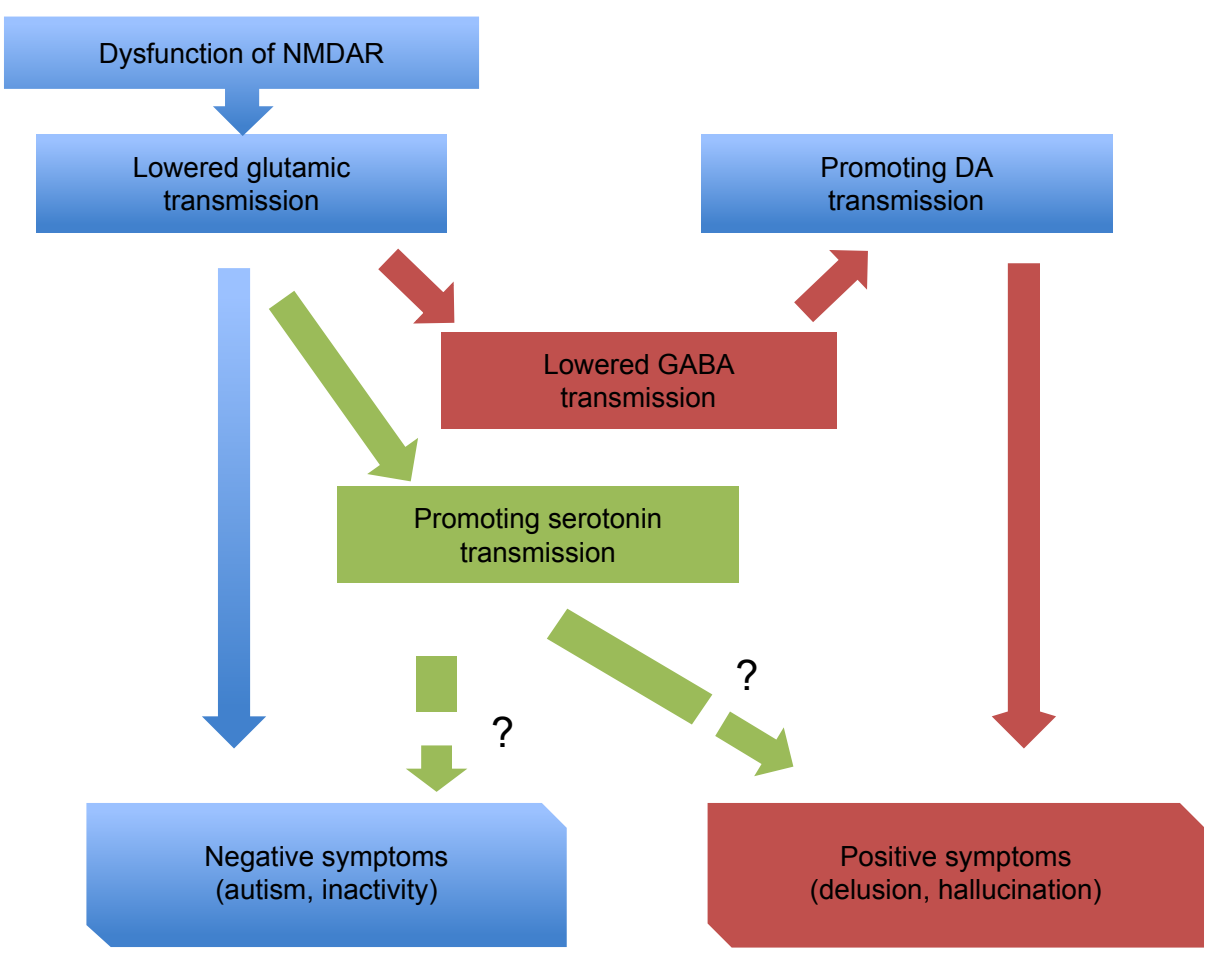

Figure 3 The schema of glutamate hypothesis of schizophrenia.

Notes: Glutamate hypothesis of schizophrenia is well known to psychiatrists. NMDAR hypofunction as a central component of the functional disconnection; this is one of the most accepted models for schizophrenia. Dysfunction of NMDAR provokes lowered glutamic transmission. As a result, many psychotic symptoms were evoked. Abbreviations: NMDAR, N-methyl-D-aspartate receptor; DA, dopamine. 
malignant syndrome. ${ }^{31,32}$ Although our current case 2 was treated well with antipsychotics, our previous several cases showed the clinical symptoms with malignant catatonia and neuroleptic malignant syndrome. ${ }^{7}$

Finally, schizophrenia is a general, heterogeneous, complicated disorder, and there are only few evidence of this pathophysiology. Referring to NMDAR hypofunction, there is established evidence with the functional disconnection of the central element; this is one of the most recognized models for schizophrenia (Figure 3). ${ }^{11,33}$ Moreover, autoimmune systems have been mentioned to be involved at least in subgroups of schizophrenia patients. Further study of antiNMDAR antibody and its related encephalitis would give essential clues for the research of schizophrenia, catatonia, and atypical psychosis.

\section{Conclusion}

For a differential diagnosis of catatonic state, it is important to detect anti-NMDAR encephalitis. This encephalitis is expected to be in remission by early detection and treatment. We should be more careful about catatonic symptoms of schizophrenia. When a patient is doubted with this encephalitis, we should screen for anti-NMDAR antibodies in CSF samples using a CBA.

\section{Acknowledgment}

We would like to thank Ms Airi Yamada for writing and editing English for this article.

\section{Disclosure}

The authors do not have conflict of interest for this paper.

\section{References}

1. Dalmau J, Tüzün E, Wu HY, et al. Paraneoplastic anti-N-methylD-aspartate receptor encephalitis associated with ovarian teratoma. Ann Neurol. 2007;61(1):25-36.

2. Iizuka T. [Clinical features and pathogenesis of anti-NMDA receptor encephalitis]. Rinsho Shinkeigaku. 2008;48(11):920-922. Japanese [with English abstract].

3. Sansing LH, Tüzün E, Ko MW, Baccon J, Lynch DR, Dalmau J. A patient with encephalitis associated with NMDA receptor antibodies. Nat Clin Pract Neurol. 2007;3(5):291-296.

4. Nokura K, Yamamoto H, Okawara Y, Koga H, Osawa H, Sakai K. Reversible limbic encephalitis caused by ovarian teratoma. Acta Neurol Scand. 1997;95(6):367-373.

5. Kleinig TJ, Thompson PD, Matar W, et al. The distinctive movement disorder of ovarian teratoma-associated encephalitis. Mov Disord. 2008; 23(9):1256-1261.

6. Iizuka T, Hara A. [Anti-NMDA receptor antibody-mediated encephalitis/ encephalopathy]. Rinsho Byori. 2009;57(3):252-261. Japanese [with English abstract].

7. Tsutsui K, Kanbayashi T, Tanaka K, et al. Anti-NMDA-receptor antibody detected in encephalitis, schizophrenia, and narcolepsy with psychotic features. BMC Psychiatry. 2012;12:37.
8. Mitsuda H. The concept of "atypical psychoses" from the aspect of clinical genetics. Acta Psychiatr Scand. 1965;41(3):372-377.

9. Dingledine R, Borges K, Bowie D, Traynelis SF. The glutamate receptor ion channels. Pharmacol Rev. 1999;51(1):7-61.

10. Cull-Candy SG, Leszkiewicz DN. Role of distinct NMDA receptor subtypes at central synapses. Sci STKE. 2004;2004(255):re16.

11. Schwartz TL, Sachdeva S, Stahl SM. Glutamate neurocircuitry: theoretical underpinnings in schizophrenia. Front Pharmacol. 2012; 3:195.

12. Hughes EG, Peng X, Gleichman AJ, et al. Cellular and synaptic mechanisms of anti-NMDA receptor encephalitis. $J$ Neurosci. 2010;30(17): $5866-5875$.

13. Dalmau J, Lancaster E, Martinez-Hernandez E, Rosenfeld MR, Balice-Gordon R. Clinical experience and laboratory investigations in patients with anti-NMDAR encephalitis. Lancet Neurol. 2011;10(1): 63-74.

14. Gleichman AJ, Spruce LA, Dalmau J, Seeholzer SH, Lynch DR. AntiNMDA receptor encephalitis antibody binding is dependent on amino acid identity of a small region within the GluN1 amino terminal domain. J Neurosci. 2012;32(32):11082-11094.

15. Li Y, Tanaka K, Wang L, Ishigaki Y, Kato N. Induction of memory deficit in mice with chronic exposure to cerebrospinal fluid from patients with anti-N-methyl-D-aspartate receptor encephalitis. Tohoku J Exp Med. 2015;237(4):329-338.

16. Abe M, Fukaya M, Yagi T, Mishina M, Watanabe M, Sakimura K. NMDA receptor GluRepsilon/NR2 subunits are essential for postsynaptic localization and protein stability of GluRzeta1/NR1 subunit. J Neurosci. 2004;24(33):7292-7304.

17. Zhang Q, Tanaka K, Sun P, et al. Suppression of synaptic plasticity by cerebrospinal fluid from anti-NMDA receptor encephalitis patients. Neurobiol Dis. 2012;45(1):610-615.

18. Titulaer MJ, McCracken L, Gabilondo I, et al. Treatment and prognostic factors for long-term outcome in patients with anti-NMDA receptor encephalitis: an observational cohort study. Lancet Neurol. 2013;12(2): 157-165.

19. Pollak TA, McCormack R, Peakman M, Nicholson TR, David AS. Prevalence of anti-N-methyl-D-aspartate (NMDA) receptor [corrected] antibodies in patients with schizophrenia and related psychoses: a systematic review and meta-analysis. Psychol Med. 2014;44(12): 2475-2487.

20. Pommepuy N, Januel D. [Catatonia: resurgence of a concept. A review of the international literature]. Encephale. 2002;28(6 Pt 1):481-492. French [with English abstract].

21. Sienaert P, Dhossche DM, Vancampfort D, De Hert M, Gazdag G. A clinical review of the treatment of catatonia. Front Psychiatry. 2014; 5:181.

22. Dalmau J, Gleichman AJ, Hughes EG, et al. Anti-NMDA-receptor encephalitis: case series and analysis of the effects of antibodies. Lancet Neurol. 2008;7(12):1091-1098.

23. Gulyayeva NA, Massie MJ, Duhamel KN. Anti-NMDA receptor encephalitis: psychiatric presentation and diagnostic challenges from psychosomatic medicine perspective. Palliat Support Care. 2014;12(2): 159-163.

24. American Psychiatric Association. Diagnostic and Statistical Manual of Mental Disorders: DSM-5. 5th ed. Washington, DC: American Psychiatric Publishing; 2013.

25. Höftberger R, Rosenfeld MR, Dalmau J. Update on neurological paraneoplastic syndromes. Curr Opin Oncol. 2015;27(6):489-495.

26. Viaccoz A, Desestret V, Ducray F, et al. Clinical specificities of adult male patients with NMDA receptor antibodies encephalitis. Neurology. 2014;82(7):556-563.

27. Gresa-Arribas N, Titulaer MJ, Torrents A, et al. Antibody titres at diagnosis and during follow-up of anti-NMDA receptor encephalitis: a retrospective study. Lancet Neurol. 2014;13(2):167-177.

28. van de Riet EH, Schieveld JN. First-onset psychosis, anti-NMDAR encephalitis, schizophrenia and consultation-liaison psychiatry. Gen Hosp Psychiatry. 2013;35(4):442-443. 
29. Steiner J, Walter M, Glanz W, et al. Increased prevalence of diverse $\mathrm{N}$-methyl-D-aspartate glutamate receptor antibodies in patients with an initial diagnosis of schizophrenia: specific relevance of IgG NR1a antibodies for distinction from N-methyl-D-aspartate glutamate receptor encephalitis. JAMA Psychiatry. 2013;70(3):271-278.

30. Lennox BR, Coles AJ, Vincent A. Antibody-mediated encephalitis: a treatable cause of schizophrenia. Br J Psychiatry. 2012;200(2): 92-94.

31. Maneta E, Garcia G. Psychiatric manifestations of anti-NMDA receptor encephalitis: neurobiological underpinnings and differential diagnostic implications. Psychosomatics. 2014;55(1):37-44.
32. Mann A, Machado NM, Liu N, Mazin AH, Silver K, Afzal KI. A multidisciplinary approach to the treatment of anti-NMDA-receptor antibody encephalitis: a case and review of the literature. J Neuropsychiatry Clin Neurosci. 2012;24(2):247-254.

33. Kanbayashi T, Tsutsui K, Tanaka K, et al. [Anti-NMDA encephalitis in psychiatry; malignant catatonia, atypical psychosis and ECT] Rinsho Shinkeigaku. 2014;54(12):1103-1106. Japanese [with English abstract].

\section{Publish your work in this journal}

Neuropsychiatric Disease and Treatment is an international, peerreviewed journal of clinical therapeutics and pharmacology focusing on concise rapid reporting of clinical or pre-clinical studies on a range of neuropsychiatric and neurological disorders. This journal is indexed on PubMed Central, the 'PsycINFO' database and CAS, and is the official journal of The International Neuropsychiatric Association (INA). The manuscript management system is completely online and includes a very quick and fair peer-review system, which is all easy to use. Visit http://www.dovepress.com/testimonials.php to read real quotes from published authors.

Submit your manuscript here: http://www.dovepress.com/neuropsychiatric-disease-and-treatment-journal 